\title{
Trajectory Planning for Spacecraft Rendezvous in Elliptical Orbits with On/Off Thrusters *
}

\author{
Rafael Vazquez* Francisco Gavilan* Eduardo F. Camacho** \\ * Departamento de Ingeniería Aeroespacial \\ ** Departamento de Ingeniería de Sistemas y Automática \\ Universidad de Sevilla, Camino de los Descubrimientos s/n, 41092, \\ Sevilla,Spain(rvazquez1@us.es,fgavilan@us.es, eduardo@esi.us.es)
}

\begin{abstract}
In a previous work, the authors developed a trajectory planning algorithm for spacecraft rendezvous which computed optimal Pulse-Width Modulated (PWM) control signals, assuming that the target was moving in a circular Keplerian orbit. In this paper we extend the algorithm to the case of an elliptical target orbit with arbitrary eccentricity. Since the orbit is elliptical, the linear time-varying Tschauner-Hempel model is used, whose exact solution is possible by using true (or eccentric) anomaly instead of time (which is directly related to both via Kepler's equation). Unlike in the circular case, computing the PWM solution itself requires numerical integration. However, explicit linearization around the computed solution turns out to be possible and is exploited for rapidly improving the solution using linear programming (LP) techniques. The algorithm is initialized by solving the impulsive problem first; the impulses are converted to PWM signals, which are used as an initial guess. Using the explicit linearization and LP, the solution is refined until a (possibly local) optimal value is reached. The efficacy of the method is shown in a simulation study where it is compared to the impulsive-only approach.
\end{abstract}

Keywords: Spacecraft autonomy, Space robotics, Pulse-width modulation, Trajectory planning, Optimal trajectory, Linearization.

\section{INTRODUCTION}

Classically, the problem of rendezvous is modeled using impulsive maneuvers; one computes a sequence of (possibly optimal) impulses (usually referred to as $\Delta V$ 's) to achieve rendezvous. Approaches based on trajectory planning and optimization (Breger and How (2008); Arzelier et al. (2013, 2011)) and predictive control (Richards and How (2003); Rossi and Lovera (2002); Asawa et al. (2006); Gavilan et al. (2009, 2012); Larsson et al. (2006)) are emerging. Some of these methods keep using impulsive maneuvers, but many allow the control signal (thrust) to take any value inside an allowed range. This type of control signal is usually referred to as Pulse-Amplitude Modulated (PAM).

However, neither impulsive actuation nor PAM actuation capture with precision the behavior of real spacecraft thrusters. A more realistic model has to take into account that, typically, thrusters are ON-OFF actuators, i.e., the thrusters are not able to produce arbitrary forces, but instead can only be switched on (producing the maximum amount of force) or off (producing no force). These switching times are the only signals that can be controlled. This type of control signal is usually referred to as Pulse-Width Modulated (PWM). Trajectory planning in the rendezvous problem with PWM actuation poses a challenge because the system becomes nonlinear in the switching times.

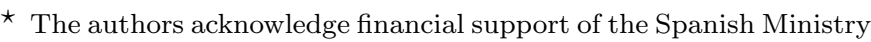
of Science and Innovation and of the European Commission for funding part of this work under grants DPI2008-05818 and EU NoE HYCON 2 (grant FP7-257462).
}

Recently, Vazquez et al. (2011) introduced a trajectory planning algorithm algorithm for spacecraft rendezvous that was able to incorporate PWM control signals. However, the result used the linear time-invariant ClohessyWiltshire model (see Clohessy and Wiltshire (1960)), which describes the relative position of the chaser spacecraft if the target is orbiting in a circular Keplerian orbit and is accurate only if the approaching vehicle is close enough to the target. In this paper we keep the proximity hypothesis but extend the result to elliptical orbits by using the Tschauner-Hempel model, which results in a linear time-varying (LTV) description of the relative position dynamics. This considerably complicates the formulation (and unfortunately increases the computation times), but the algorithm is essentially the same; it uses linearization around reference values to faithfully capture the dependence of the system on small variations of the switching times (at least close to the reference values) and then optimizes these variations iteratively. We use a formulation based on eccentric anomaly as a convenient way to write down the explicit evolution of the system. However, unlike in the circular case, we are not able to explicitly write the transition matrices when using PWM inputs, and have to numerically compute the system evolution. We are able, though, to explicitly compute the linearization for the system around the PWM solution, which allows using linear programming (LP), at least for small variations. Thus the resulting algorithm is less explicit than in the circular case, but the resulting optimization subproblems are still explicit and linear. An initial guess for the linearization is obtained by solving the problem using impulsive control 
signals (which is another difference with Vazquez et al. (2011), that used PAM actuation for this first iteration; this modification allows explicit initialization). The impulses are then converted to PWM signals and used as initialization for our algorithm. Iterating, the solution is refined until an optimal value is reached. The algorithm is simple and reasonably fast, and we show simulations of its application comparing it with an impulsive-only approach.

The structure of the paper is as follows. In Section 2 we introduce the Tschauner-Hempel model, both in the impulsive and PWM case. We follow with Section 3 where we formulate the underlying optimization problems. Section 4 describes a method that solves the planning problem using PWM signals. In Section 5 we show simulations of the method. We finish with some remarks in Section 6 .

\section{TSCHAUNER-HEMPEL MODEL OF SPACECRAFT RENDEZVOUS}

The Tschauner-Hempel model (see Tschauner and Hempel (1965) or Carter (1998)) assumes that the target vehicle is passive and moving along an elliptical orbit with semimajor axis $a$ and eccentricity $e$. The system equations are LTV and cannot be exactly integrated in time to obtain a discrete transition model; however, if we substitute the time $t$ by the eccentric anomaly of the target orbit, $E$, we can obtain explicit expressions for the system evolution in the impulsive case (but, however, only for the out-of-plane evolution in the PWM case).

Let us first establish some notation. Note that $t$ and $E$ are related in a one-to-one fashion by using Kepler's equation:

$$
n\left(t-t_{p}\right)=E-e \sin E,
$$

where $t_{p}$ is the time at periapsis which we use as a starting point to measure the eccentric anomaly $E$ (we choose this time such that it is equal or less than the starting time which we denote as $\left.t_{0}\right)$. This equation is readily invertible (see any Orbital Mechanics reference, such as Wie (1998)), and we will represent its inverse by the function $K$, i.e. $E=K(t)$. Denote by $E_{0}$ the true anomaly corresponding to $t_{0}$, this is, $E_{0}=K\left(t_{0}\right)$ and $E_{k}=K\left(t_{k}\right)=K\left(t_{0}+\right.$ $k T)$, where $T$ is an adequately chosen sampling time. Call as $x_{k}, y_{k}$, and $z_{k}$ the position of the chaser in a localvertical/local-horizontal (LVLH) frame of reference fixed on the center of gravity of the target vehicle at time $t_{k}$. In the (elliptical) LVLH frame, $x$ refers to the radial position, $z$ to the out-of-plane position (in the direction of the orbital angular momentum), and $y$ is perpendicular to these coordinates (no longer aligned with the target velocity given that its orbit is not circular). The velocity and inputs of the chaser in the LVLH frame at time $t_{k}$ are denoted, respectively, by $v_{x, k}, v_{y, k}$, and $v_{z, k}$, and by $u_{x, k}$, $u_{y, k}$, and $u_{z, k}$.

If there is no actuation (i.e. $u_{x, k}=u_{y, k}=u_{z, k}=0$ ), the resulting transition equation was obtained in a simple form in Yamanaka and Ankersen (2002) as follows:

$$
\mathbf{x}_{k+1}=A\left(t_{k+1}, t_{k}\right) \mathbf{x}_{k}
$$

where

$$
\mathbf{x}_{k}=\left[\begin{array}{llllll}
x_{k} & y_{k} & z_{k} & v_{x, k} & v_{y, k} & v_{z, k}
\end{array}\right]^{T},
$$

and where $A\left(t_{k+1}, t_{k}\right)=Y_{K\left(t_{k+1}\right)} Y_{K\left(t_{k}\right)}^{-1}$, with $Y_{t_{k}}$ being the fundamental matrix solution of the Tschauner-Hempel model, which are expressed in Yamanaka and Ankersen
(2002) as a function of true anomaly $\theta$. However there is a one-to-one relation between $E$ and $\theta$ given by

$$
\tan \frac{\theta}{2}=\sqrt{\frac{1+e}{1-e}} \tan \frac{E}{2},
$$

which we exploit in the sequel. The explicit expression of the matrices ${ }^{1}$ is found in (5) and (6), where the following symbols are used (expressed in terms of $E$ ):

$$
\begin{aligned}
& \rho=\frac{1-e^{2}}{1-e \cos E_{k}}, s=\frac{\sqrt{1-e^{2}} \sin E_{k}}{1-e \cos E_{k}}, c=\frac{\cos E_{k}-e}{1-e \cos E_{k}},(7) \\
& J=\frac{E_{k}-E_{k-1}-e\left(\sin E_{k}-\sin E_{k-1}\right)}{\left(1-e^{2}\right)^{3 / 2}}, \alpha=\frac{n}{\left(1-e^{2}\right)^{3 / 2}} .(8)
\end{aligned}
$$

Next, we formulate two versions of the discretized equations. In the first version, the control inputs are considered impulses which are applied at the middle of the sampling interval. This is referred to as the impulsive discrete model. In a second, more realistic version, thrusters can only be switched on (producing the maximum force) or off (producing no force), and only once during each sampling time. This is referred to as the PWM discrete model.

\subsection{Impulsive discrete model}

For the impulsive model, we assume that we can apply an impulse $u$ in any axis and at any given sample time. For simplicity's purpose, we assume that only one impulse per axis is allowed at each time interval and model the impulse at the beginning of the time interval. Exploiting the linearity of the system, it can be easily shown that

$$
\mathbf{x}_{k+1}=A\left(t_{k+1}, t_{k}\right) \mathbf{x}_{k}+B\left(t_{k+1}, t_{k}\right) \mathbf{u}_{k},
$$

where $\mathbf{u}_{k}=\left[\begin{array}{lll}u_{x, k} & u_{y, k} & u_{z, k}\end{array}\right]^{T}$ and

$$
B\left(t_{k+1}, t_{k}\right)=A\left(t_{k}+1, t_{k}\right)\left[\begin{array}{lll}
0 & 0 & 0 \\
0 & 0 & 0 \\
0 & 0 & 0 \\
1 & 0 & 0 \\
0 & 1 & 0 \\
0 & 0 & 1
\end{array}\right] .
$$

\section{Compact formulation}

Next we develop a compact formulation that simplifies the notation of the problem. The state at time $t_{k+1}$, given the initial state at time $t_{0}$ (which is denoted as $\mathbf{x}_{0}$ ) and the input signals from $t_{0}$ to time $t_{k}$, is computed by applying recursively Equation (9) and using the fact that $A\left(t_{i+1}, t_{i}\right) A\left(t_{i}, t_{i-1}\right)=A\left(t_{i+1}, t_{i-1}\right)$ :

$\mathbf{x}_{k+1}=A\left(t_{k+1}, t_{0}\right) \mathbf{x}_{0}+\sum_{j=0}^{k} A\left(t_{k+1}, t_{j+1}\right) B\left(t_{j+1}, t_{j}\right) \mathbf{u}_{j}$,

where it must be noted that $A\left(t_{i}, t_{i}\right)=\mathrm{Id}$, where Id is the identity matrix. Define now $\mathbf{x}_{\mathbf{S}}$ and $\mathbf{u}_{\mathbf{S}}$ as a stack of $N_{p}$ states and input signals, respectively, spanning from time $t_{1}$ to time $t_{N_{p}}$, where $N_{p}$ is the planning horizon:

$$
\mathbf{x}_{\mathbf{S}}=\left[\begin{array}{c}
\mathbf{x}_{1} \\
\mathbf{x}_{2} \\
\vdots \\
\mathbf{x}_{N_{p}}
\end{array}\right], \mathbf{u}_{\mathbf{S}}=\left[\begin{array}{c}
\mathbf{u}_{0} \\
\mathbf{u}_{1} \\
\vdots \\
\mathbf{u}_{N_{p}-1}
\end{array}\right]
$$

1 Our expressions slightly differ from Yamanaka and Ankersen (2002) given that we premultiply the two transformation matrices that appear in that paper; also, our reference axes are not the same. 


$$
\begin{aligned}
& Y_{E_{k}}=\left[\begin{array}{cccccc}
s & 0 & 0 & 2 / \rho-3 e s J & -c & 0 \\
c(1+1 / \rho) & 1 / \rho & 0 & -3 J s(1+\rho) & 0 & \\
0 & 0 & c / \rho & 0 & 0 & s / \rho \\
\alpha \rho^{2} c & 0 & 0 & \alpha\left(-e s-3 e \rho^{2} J c\right) & \alpha \rho^{2} s & 0 \\
\alpha s\left(-1-\rho^{2}\right) & \alpha e s & 0 & \alpha \rho(3 e s \rho J-3) & \alpha\left(c+e+c \rho^{2}\right) & 0 \\
0 & 0 & -s \alpha & 0 & 0 & (c+e) \alpha
\end{array}\right], \\
& Y_{E_{k-1}}^{-1}=\frac{1}{\left(1-e^{2}\right)}\left[\begin{array}{cccccc}
-s\left(\rho^{2}+2 \rho+e^{2}\right) & e s^{2}(1+\rho) & 0 & \frac{c-2 e / \rho}{\alpha} & -s \frac{1+1 / \rho}{\alpha} & 0 \\
-e s(1+\rho)^{2} & \rho^{2}(1-c e)+e^{2} s^{2} & 0 & \frac{e c-2 / \rho}{\alpha} & -e s \frac{1+1 / \rho}{\alpha} & 0 \\
0 & 0 & (c+e)\left(1-e^{2}\right) & 0 & 0 & \frac{-s\left(1-e^{2}\right)}{\alpha \rho} \\
\rho^{2}(1+\rho) & -e s \rho^{2} & 0 & \frac{e s}{\alpha} & \frac{\rho}{\alpha} & 0 \\
3 \rho(c+e)-e \rho s^{2} & -e s c(1+\rho)-e^{2} s & 0 & \frac{s}{\alpha} & \frac{c}{\alpha}(1+1 / \rho)+\frac{e}{\alpha \rho} & 0 \\
0 & 0 & s\left(1-e^{2}\right) & 0 & 0 & \frac{c\left(1-e^{2}\right)}{\alpha \rho}
\end{array}\right] .(6)
\end{aligned}
$$

Then,

$$
\mathbf{x}_{\mathbf{S}}=\left[\begin{array}{c}
A\left(t_{1}, t_{0}\right) \mathbf{x}_{0}+B\left(t_{1}, t_{0}\right) \mathbf{u}_{0} \\
A\left(t_{2}, t_{0}\right) \mathbf{x}_{0}+\sum_{j=0}^{1} A\left(t_{2}, t_{j+1}\right) B\left(t_{j+1}, t_{j}\right) \mathbf{u}_{j} \\
\vdots \\
A\left(t_{N_{p}}, t_{0}\right) \mathbf{x}_{0}+\sum_{j=0}^{N_{p}-1} A\left(t_{N_{p}}, t_{j+1}\right) B\left(t_{j+1}, t_{j}\right) \mathbf{u}_{j}
\end{array}\right],
$$

which can be written as

$$
\mathbf{x}_{\mathbf{S}}=\mathbf{F} \mathbf{x}_{0}+\mathbf{G} \mathbf{u}_{\mathbf{S}}
$$

where $\mathbf{G}$ is a block lower triangular matrix with its nonnull elements defined by $(\mathbf{G})_{i j}=A\left(t_{i}, t_{j}\right) B\left(t_{j}, t_{j-1}\right)$ and the matrix $\mathbf{F}$ is defined as:

$$
\mathbf{F}=\left[\begin{array}{c}
A\left(t_{1}, t_{0}\right) \\
\vdots \\
A\left(t_{N_{p}}, t_{0}\right)
\end{array}\right] .
$$

\subsection{PWM discrete formulation}

Consider now ON-OFF thrusters which are not able to produce any value of force, but can be only switched on or off. We assume that there is an aligned pair of thrusters for each direction $i=1,2,3$ with opposing orientation. To distinguish between the positive and negative they are denoted as $u_{i}^{+}$and $u_{i}^{-}$, whereas the maximum thrust is referred to as $\bar{u}_{i}^{+}$and $\bar{u}_{i}^{-}$, respectively. Finally, during each sample time each thruster is allowed to fire only once.

Thus, the PWM output for each time interval $k$ is completely described by two new control variables for each pair of thrusters, as shown in Fig. 1: the pulse width $\kappa_{i, k}^{+}$ and the pulse start time $\tau_{i, k}^{+}$(for the positively oriented thruster in the direction $i$ ) and similarly $\kappa_{i, k}^{-}$and $\tau_{i, k}^{-}$for the negatively oriented thruster in the direction $i$. Then, for $t \in[k T,(k+1) T]$, we have:

$$
u_{i}^{+}(t)= \begin{cases}0, & t \in\left[k T, k T+\tau_{i, k}^{+}\right], \\ \bar{u}_{i}^{+}, & t \in\left[k T+\tau_{i, k}^{+}, k T+\tau_{i, k}^{+}+\kappa_{i, k}^{+}\right], \\ 0, & t \in\left[k T+\tau_{i, k}^{+}+\kappa_{i, k}^{+},(k+1) T\right],\end{cases}
$$

and similarly for the negatively oriented thrusters. The new variables control variables verify $\kappa_{i, k}^{+}>0, \tau_{i, k}^{+}>0$ and $\tau_{i, k}^{+}+\kappa_{i, k}^{+}<T$, and similarly for the negatively oriented thrusters. The last constraint prevent the PWM signal to spill over to the next time interval.

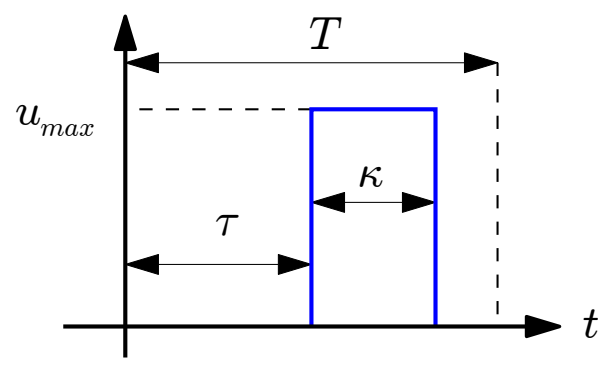

Fig. 1. PWM Variables.

Call the PWM control variables at $t_{k}$ as $\mathbf{u}_{k}^{P}$ :

$\mathbf{u}_{k}^{P}=\left[\tau_{1, k}^{+} \kappa_{1, k}^{+} \tau_{1, k}^{-} \kappa_{1, k}^{-} \tau_{2, k}^{+} \kappa_{2, k}^{+} \tau_{2, k}^{-} \kappa_{2, k}^{-} \tau_{3, k}^{+} \kappa_{3, k}^{+} \tau_{3, k}^{-} \kappa_{3, k}^{-}\right]^{T}$.

To find the state transition equations for PWM inputs, define, for $i=1,2,3$,

$$
b_{i}(t, \tau, \kappa)=\int_{t+\tau}^{t+\tau+\kappa} Y_{K(s)}^{-1} C_{i+3} d s,
$$

where $C_{i}$ is a column vector of zeros with a value of one at row $i$. This is obtained from the variation of parameters formula for a linear inhomogeneous time-varying system. This equation can be expressed in terms of eccentric anomaly as follows:

$$
b_{i}(t, \tau, \kappa)=\int_{K(t+\tau)}^{K(t+\tau+\kappa)} Y_{E}^{-1} C_{i+3} \frac{1-e \cos E}{n} d E .
$$

However we cannot explicitly compute these integrals, due to the fact that the expression for $Y^{-1}$ when $E \neq E_{k-1}$ is more complex than (6) due to the fact that the $J$ term from $Y$ in (5) should also appear in the inverse; it does not appear in the homogeneous case due to choosing the lower limit in the integral in (8) as the value of $E$ in the previous time step. Only $b_{3}$ is analytically computable, resulting in 


$$
b_{3}=\frac{1}{n \kappa}\left[\begin{array}{llllll}
0 & 0 & f_{1} & 0 & 0 & f_{2}
\end{array}\right]^{T},
$$

where

$$
f_{1}=\sqrt{1-e^{2}}\left[\frac{\cos E-e / 4\left(\cos ^{2} E-\sin ^{2} E\right)}{1-e^{2}}\right]_{K(t+\tau)}^{K(t+\tau+\kappa)},
$$

$f_{2}=\frac{f_{3}}{1-e^{2}}+\frac{1}{1-e^{2}}\left[e^{2} \sin E-e \frac{E}{2}-e \frac{\sin 2 E}{4}\right]_{K(t+\tau)}^{K(t+\tau+\kappa)},(21)$

$f_{3}=[\sin E-e E]_{K(t+\tau)}^{K(t+\tau+\kappa)}$.

Then the system evolution equation for the PWM case is

$$
\mathbf{x}_{k+1}=A\left(t_{k+1}, t_{k}\right) \mathbf{x}_{k}+B_{P W M}\left(t_{k+1}, t_{k}, \mathbf{u}_{k}^{P}\right),
$$

where

$$
B_{P W M}=\sum_{i=1}^{i=3} B_{i}^{+} \bar{u}_{i}^{+}+\sum_{i=1}^{i=3} B_{i}^{-} \bar{u}_{i}^{-} .
$$

with $B_{i}^{ \pm}$column vectors defined by

$$
B_{i}^{ \pm}\left(t_{k+1}, t_{k}, \mathbf{u}_{k}^{P}\right)=Y\left(t_{k+1}\right) b_{i}\left(t, \tau_{i, k}^{ \pm}, \kappa_{i, k}^{ \pm}\right)
$$

In this equation we need to compute $b_{1}$ and $b_{2}$ numerically. Compact formulation

The compact formulation developed before can be readily adapted to PWM inputs. Equation (13) is now written as

$$
\mathbf{x}_{\mathbf{S}}=\mathbf{F} \mathbf{x}_{0}+\mathbf{G}_{\mathbf{P W M}}\left(\mathbf{u}_{\mathbf{S}}^{\mathbf{P}}\right)
$$

where $\mathbf{u}_{\mathbf{S}}^{\mathbf{P}}$ is a stack vector with all the PWM signals,

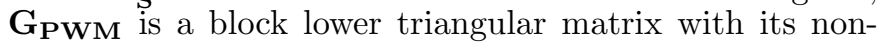
null elements defined

\section{FORMULATION OF THE PLANNING PROBLEM}

Next we formulate our planning problem, introducing the constraints and the objective function. The formulation is done for both impulsive and PWM control signals.

\subsection{Constraints on the problem}

Inequality constraints on the state For sensing purposes (see e.g. Breger and How (2008)), during rendezvous it is required that the chaser vehicle remains inside a line of sight (LOS) area. To simplify the constraint, we consider a 2-D LOS area as shown in Figure 2.

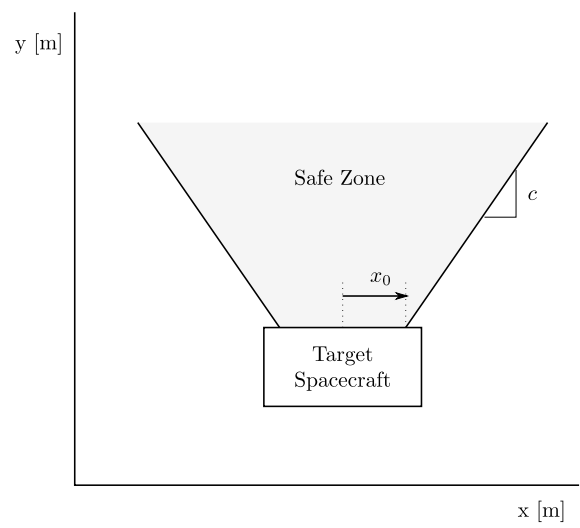

Fig. 2. Line of Sight region.
For impulsive control these constraints can be formulated (see Vazquez et al. (2011)) as

$$
\mathbf{A}_{c} \mathbf{G u}_{\mathbf{S}} \leq \mathbf{b}_{c}-\mathbf{A}_{c} \mathbf{F} \mathbf{x}_{0}
$$

and similarly for the case of PWM control.

\section{Equality constraints on the state}

Equality constraints are formulated to ensure that the chaser spacecraft arrives at the origin with zero velocity at the end of the planning horizon. Thus, these constraints can be written as $\mathbf{x}\left(N_{p}\right)=\mathbf{0}$.

Following Vazquez et al. (2011), we formulate the arrival conditions as equality constraints for the control signals:

$$
\mathbf{A}_{e q} \mathbf{G u}_{\mathbf{S}}=-\mathbf{A}_{e q} \mathbf{F} \mathbf{x}_{0}
$$

and similarly for the case of PWM control.

\section{Input constraints}

For the case of impulsive control, we consider limitations on the magnitude of the impulses. To be able to convert the impulsive signals to PWM signals, we fix the maximum (resp. minimum) of the impulse as the maximum (resp. minus minimum) thrust of the PWM actuator times the sample time.

$$
-T \overline{\mathbf{u}}^{-} \leq \mathbf{u}_{\mathbf{S}} \leq T \overline{\mathbf{u}}^{+}
$$

For the case of PWM control, the constraints are given by $\kappa_{i}^{ \pm}(k)>0, \tau_{i}^{ \pm}(k)>0$ and $\tau_{i}^{ \pm}(k)+\kappa_{i}^{ \pm}(k)<T$.

\subsection{Objective function}

The objective function to be minimized in the planning problem is the 1-norm of the control signal, which is proportional to fuel consumption.

\section{Impulsive control inputs}

For the case of impulsive control inputs, the selected control function is given by:

$$
J_{I M P}=\sum_{k=0}^{N_{p}-1}\left\|\mathbf{u}_{k}\right\|_{1}=\left\|\mathbf{u}_{\mathbf{S}}\right\|_{1} .
$$

\section{PWM control inputs}

For the case of PAM control inputs, using (15) it can be seen that the selected objective function is given by:

$$
J_{P W M}=\sum_{k=0}^{N_{p}-1} \sum_{i=1}^{3}\left(\bar{u}_{i}^{+} \kappa_{i, k}^{+}+\bar{u}_{i}^{-} \kappa_{i, k}^{-}\right) .
$$

\section{COMPUTATION OF THE OPTIMAL CONTROL INPUT}

As seen in Section 2.2, the plant equations in the PWM case are nonlinear in the switching times. To find the optimal control input without needing to solve a nonlinear planning problem, the following scheme is proposed:

Step 1. The impulsive optimization problem is solved. Step 2. The impulsive signals are converted to PWM inputs.

Step 3. The trajectory of the system with the PWM inputs is computed numerically.

Step 4. The system with PWM inputs is linearized around the previous step solution, thus obtaining a linear, explicit plant with respect to increments in 
the PWM inputs. An LP is then posed and solved to optimize the increments.

Step 5. The resulting solution is used to improve the approximation towards the real solution. Repeat the computation of Step 4 and the linearization process of Step 3 around the new solution. The process is iterated until the solution converges or time is up.

Next, we describe all the steps in our scheme.

\subsection{Computation of impulsive control input}

To compute the optimal control plan (with impulsive control signals), one solves

$$
\min _{\mathbf{u}_{\mathbf{S}}} \quad J_{I M P}\left(\mathbf{u}_{\mathbf{S}}\right)
$$

subject to: $\mathbf{A}_{c} \mathbf{G u}_{\mathbf{S}} \leq \mathbf{b}_{c}-\mathbf{A}_{c} \mathbf{F} \mathbf{x}_{\mathbf{0}}$

$$
\begin{gathered}
\mathbf{A}_{e q} \mathbf{G} \mathbf{u}_{\mathbf{S}}=-\mathbf{A}_{e q} \mathbf{F} \mathbf{x}_{0} \\
-T \overline{\mathbf{u}}^{-} \leq \mathbf{u}_{\mathbf{S}} \leq T \overline{\mathbf{u}}^{+} .
\end{gathered}
$$

Since the cost function and the constraints are linear, then (32) can be readily solved.

\subsection{Initial PWM solution: Adapting the impulsive solution}

The impulsive solution from (32) is transformed to a PWM inputs, for each time instant $k$ and direction $i$, as follows:

(1) Use the positive or negative thruster according to the sign of $u_{i, k}$.

(2) The pulse width has an area equal to the impulse value: $\kappa_{i, k}^{ \pm}=\frac{\left|u_{i, k}\right|}{\bar{u}_{i}^{ \pm}}$, where $\bar{u}_{i}^{ \pm}$is the maximum level of the (positive or negative) thruster $i$.

(3) Since the impulse was modeled to start at the beginning of a time sample, $\tau_{i, k}^{ \pm}=0$.

The PWM signals $\mathbf{u}_{k}^{P}$ constructed by this method produce a similar output to the system driven by impulsive signals. However, the PWM results are not necessarily optimal since their constraints are quite different; in fact, they might not even verify the constraints (as we will see in simulations). Thus, this solution is only used as an initialization for the optimization algorithm proposed next.

\subsection{Computation of trajectories under PWM inputs}

Next we apply (26) to compute the output of the system with PWM inputs. Note that $\mathbf{G}_{\mathbf{P W M}}\left(\mathbf{u}_{\mathbf{S}}^{\mathbf{P}}\right)$ has to be computed numerically because it contains the term $B_{P W M}\left(t_{k+1}, t_{k}, \mathbf{u}_{k}^{P}\right)$, which couldn't be obtained explicitly due to the integral (18).

\subsection{Refined PWM solution: An optimization algorithm}

Following Vazquez et al. (2011), we approximate (23) by linearizing $B_{P W M}$ around $\mathbf{u}_{k}^{P}$. Then, we obtain

$$
\begin{aligned}
\mathbf{x}_{k+1}= & A\left(t_{k+1}, t_{k}\right) \mathbf{x}_{k}+B_{P W M}\left(t_{k+1}, t_{k}, \mathbf{u}_{k}^{P}\right) \\
& +B^{\Delta}\left(t_{k+1}, t_{k}, \mathbf{u}_{k}^{P}\right) \boldsymbol{\Delta} \mathbf{u}_{k}^{P},
\end{aligned}
$$

where $B^{\Delta}$ is a matrix computed as

$$
\left(B^{\Delta}\right)_{i, j}=\frac{\partial\left(B_{P W M}\left(t_{k+1}, t_{k}, \mathbf{u}_{k}^{P}\right)\right)_{i}}{\partial\left(\mathbf{u}_{k}^{P}\right)_{j}},
$$

The variable $\boldsymbol{\Delta} \mathbf{u}_{k}^{P}$ represents the increments or decrements with respect to $\mathbf{u}_{k}^{P}$. Using the fact that $B_{P W M}$ is defined in (24) by using the $B_{i}$ 's in (25), with the $b_{i}$ 's defined in (17), notice that

$$
B_{i}\left(t_{k+1}, t_{k}, \tau, \kappa\right)=Y\left(t_{k+1}\right) \int_{t_{k}+\tau}^{t_{k}+\tau+\kappa} Y_{K(s)}^{-1} C_{i+3} \bar{u}_{i} d s
$$

therefore

$$
\begin{aligned}
\frac{\partial}{\partial \tau} B_{i}\left(t_{k+1}, t_{k}, \tau, \kappa\right)= & A\left(t_{k+1}, t_{k}+\tau+\kappa\right) C_{i+3} \bar{u}_{i} \\
& -A\left(t_{k+1}, t_{k}+\tau\right) C_{i+3} \bar{u}_{i} \\
\frac{\partial}{\partial \kappa} B_{i}\left(t_{k+1}, t_{k}, \tau, \kappa\right)= & A\left(t_{k+1}, t_{k}+\tau+\kappa\right) C_{i+3} \bar{u}_{i},
\end{aligned}
$$

and thus we can compute $B^{\Delta}$ explicitly from $Y$ and $Y^{-1}$, which is important not only for speed but also for being able to pose an explicit LP. Equation (33) becomes

$$
\mathbf{x}_{\mathbf{S}}=\mathbf{F} \mathbf{x}_{0}+\mathbf{G}_{\mathbf{P W M}}\left(\mathbf{u}_{\mathbf{S}}^{\mathbf{P}}\right)+\mathbf{G}_{\boldsymbol{\Delta}}\left(\mathbf{u}_{\mathbf{S}}^{\mathbf{P}}\right) \boldsymbol{\Delta}_{\mathbf{S}},
$$

where $\mathbf{G}_{\boldsymbol{\Delta}}\left(\mathbf{u}_{\mathbf{S}}^{\mathbf{P}}\right)$ is a block lower triangular matrix with its non-null elements defined by

$$
\left(\mathbf{G}_{\boldsymbol{\Delta}}\left(\mathbf{u}_{\mathbf{S}}^{\mathbf{P}}\right)\right)_{i j}=A\left(t_{i}, t_{j}\right) B^{\Delta}\left(t_{j}, t_{j-1}, \mathbf{u}_{\mathbf{j}-\mathbf{1}}^{\mathbf{P}}\right),
$$

and $\boldsymbol{\Delta}_{\mathbf{S}}$ is a stack vector of the increment in the PWM variables $\boldsymbol{\Delta} \mathbf{u}_{k}^{P}$. The LOS constraints (27) become

$$
\mathbf{A}_{c} \mathbf{G}_{\boldsymbol{\Delta}} \boldsymbol{\Delta}_{\mathbf{S}} \leq \mathbf{b}_{c}-\mathbf{A}_{c} \mathbf{F} \mathbf{x}_{0}-\mathbf{A}_{c} \mathbf{G}_{\mathbf{P W M}}
$$

where the dependence of $\mathbf{G}_{\mathbf{P W M}}$ and $\mathbf{G}_{\boldsymbol{\Delta}}$ on $\mathbf{u}_{\mathbf{S}}^{\mathbf{P}}$ has been omitted. Similarly, the equality constraints become:

$$
\mathbf{A}_{e q} \mathbf{G}_{\boldsymbol{\Delta}} \boldsymbol{\Delta}_{\mathbf{S}}=-\mathbf{A}_{e q} \mathbf{F} \mathbf{x}_{0}-\mathbf{A}_{e q} \mathbf{G}_{\mathbf{P W M}}
$$

The constraints on the $\boldsymbol{\Delta} \mathbf{u}_{k}^{P}$ are as follows:

$$
\begin{aligned}
-\Delta \kappa_{i}^{ \pm}(k) & \leq \kappa_{i}^{ \pm}(k),-\Delta \tau_{i}^{ \pm}(k) \leq \tau_{i}^{ \pm}(k) \\
\Delta \tau_{i}^{ \pm}(k)+\Delta \kappa_{i}^{ \pm}(k) & \leq T-\tau_{i}^{ \pm}(k)-\kappa_{i}^{ \pm}(k), \\
\left|\boldsymbol{\Delta} \mathbf{u}_{k}^{P}\right| & \leq \Delta^{M A X},
\end{aligned}
$$

where (43) is used to avoid large variations that might make the linearization approximation to fail. These constraints are summarized as $\mathbf{A}_{\boldsymbol{\Delta}}(k) \boldsymbol{\Delta}_{\mathbf{S}}(k) \leq \mathbf{b}_{\boldsymbol{\Delta}}(k)$. Finally, the objective function can be rewritten in terms of $\boldsymbol{\Delta}_{\mathbf{S}}$ as $J\left(\mathbf{u}_{\mathbf{S}}^{\mathbf{P}}, \boldsymbol{\Delta}_{\mathbf{S}}\right)=J_{P W M}\left(\mathbf{u}_{\mathbf{S}}^{\mathbf{P}}\right)+J^{\Delta}\left(\boldsymbol{\Delta}_{\mathbf{S}}\right)$, where

$$
J^{\Delta}\left(\boldsymbol{\Delta}_{\mathbf{S}}\right)=\sum_{k=0}^{N_{p}-1} \sum_{i=1}^{3}\left(\bar{u}_{i}^{+} \Delta \kappa_{i, k}^{+}+\bar{u}_{i}^{-} \Delta \kappa_{i, k}^{-}\right) .
$$

Thus, an LP with PWM outputs can be posed as follows:

$$
\begin{aligned}
\min _{\boldsymbol{\Delta}_{\mathbf{S}}} & J^{\Delta}\left(\boldsymbol{\Delta}_{\mathbf{S}}\right) \\
\text { s. t.: } & \mathbf{A}_{c} \mathbf{G}_{\boldsymbol{\Delta}} \boldsymbol{\Delta}_{\mathbf{S}} \leq \mathbf{b}_{c}-\mathbf{A}_{c} \mathbf{F} \mathbf{x}_{0}-\mathbf{A}_{c} \mathbf{G}_{\mathbf{P W M}}, \\
\mathbf{A}_{e q} \mathbf{G}_{\boldsymbol{\Delta}} \boldsymbol{\Delta}_{\mathbf{S}} & =-\mathbf{A}_{e q} \mathbf{F} \mathbf{x}_{0}-\mathbf{A}_{e q} \mathbf{G}_{\mathbf{P W M}}, \\
& \mathbf{A}_{\boldsymbol{\Delta}} \boldsymbol{\Delta}_{\mathbf{S}} \leq \mathbf{b}_{\boldsymbol{\Delta}} .
\end{aligned}
$$

The solution $\boldsymbol{\Delta}_{\mathbf{S}}$ is used to recompute new PWM inputs, $\mathbf{u}_{\mathbf{S}}^{\mathbf{P}^{\mathbf{N E W}}}=\mathbf{u}_{\mathbf{S}}^{\mathbf{P}}+\boldsymbol{\Delta}_{\mathbf{S}}$. Then $\mathbf{u}_{\mathbf{S}}^{\mathbf{P}^{\mathbf{N E W}}}$ is used to recompute the matrices in (45), including $\mathbf{G}_{\mathbf{P W M}}$, and the optimization problem is solved again. The procedure is iterated until there is convergence, as shown next in simulations.

\section{SIMULATION RESULTS}

For numerical simulations we choose $N_{p}=50$ as planning horizon, $T=60 \mathrm{~s}$, and $\bar{u}=10^{-1} \mathrm{~N} / \mathrm{kg}$. The target orbit 

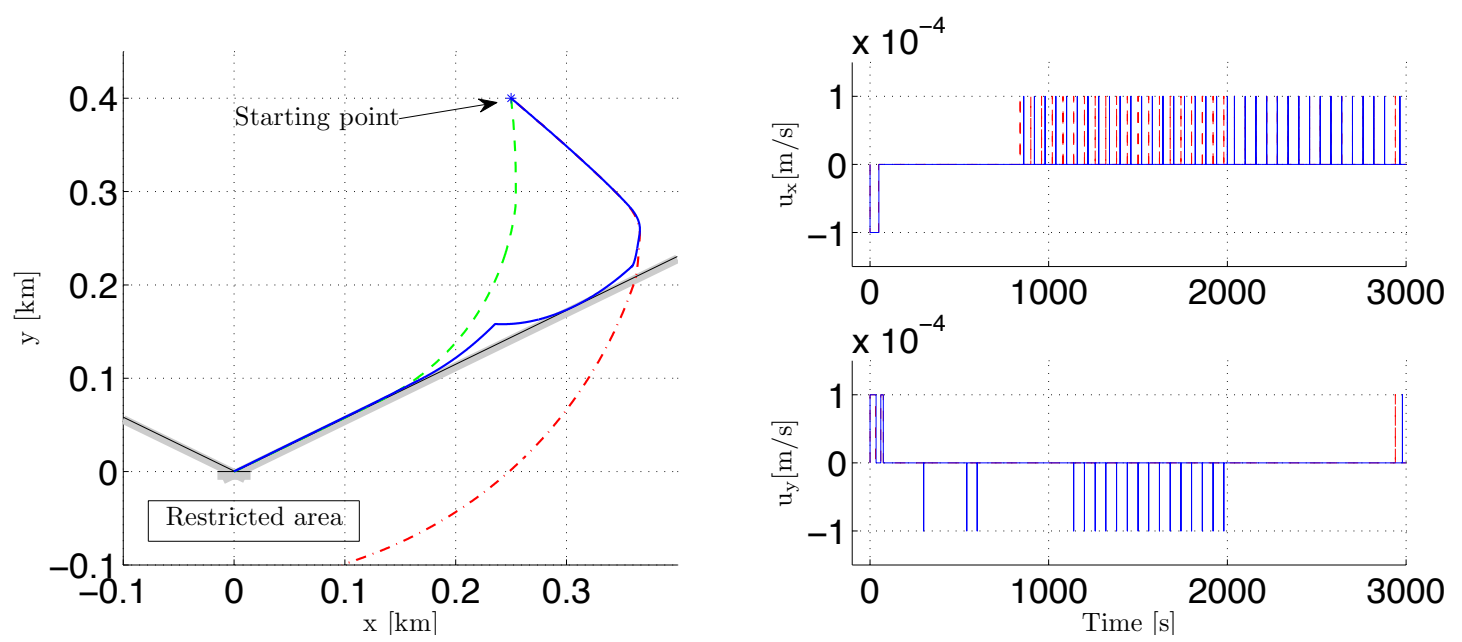

Fig. 3. Left: System trajectories in the target orbital plane: with impulsive inputs (dashed), PWM inputs computed from impulsive solution (dot-dashed), and final computed PWM solution (solid). Right: PWM inputs $\left(u_{x}\right.$ and $\left.u_{y}\right)$ for impulsive solution (dot-dashed) and final computed solution (solid).

has $e=0.7$ and perigee altitude $h_{p}=500 \mathrm{~km}$. Initials conditions were $\theta_{0}=45^{\circ}, \mathbf{r}_{0}=\left[\begin{array}{lll}0.25 & 0.4-0.2\end{array}\right]^{T} \mathrm{~km}, \mathbf{v}_{0}=$ $[0.005-0.005-0.005]^{T} \mathrm{~km} / \mathrm{s}$. The LOS constraint is defined by $x_{0}=0.001 \mathrm{~km}$ and $C_{L O S}=\tan 30^{\circ}$.

A projection on the orbital plane of the solution trajectory and its required thrust profile are shown in Fig. 3 (left and right, respectively) together with the ideal impulsive approximation and its corresponding PWM realization. It can be seen that the PWM solution obtained from the ideal solution does not verify the constraints nor does it rendezvous with the target. The algorithm required 6 iterations, but the solution after the first iteration was already quite good. The cost for the impulsive solution was $14.6 \mathrm{~m} / \mathrm{s}$, while the last PWM iteration had a cost of $15.5 \mathrm{~m} / \mathrm{s}$; it seems logical that going from an idealized (impulsive) model to a more realistic one would imply a higher cost and a less direct trajectory, given that the velocity cannot be instantly changed. Each iteration took about one second on a conventional computer, using MATLAB and the open-source lpsolve package (see Berkelaar et al. (2010)) for numerical optimization. A comparison between the initial (impulsive) and final PWM inputs (Fig. 3, right) shows that, for both, most of the effort is spent at the beginning, with some mid-course and final corrections with different timings for both cases.

\section{CONCLUDING REMARKS}

We have presented a method to compute optimal PWM inputs for the problem of rendezvous in elliptical orbits. The algorithm might be particularly useful for satellites with small specific thrust. Our approach is semi-explicit, and uses numerical integration and explicit linear optimization. We have included LOS and terminal constraints, but other constraints such as fail-tolerant or safety restrictions can be included. Its use in a receding horizon scheme seems feasible, particularly if a faster method to compute the PWM solution is found.

\section{REFERENCES}

Arzelier, D., Kara-Zaitri, M., Louembet, C., and Delibasi, A. (2011). Using polynomial optimization to solve the fuel-optimal linear impulsive rendezvous problem. $J$. Guid. Contr. Dynam., 34, 1567-1572.

Arzelier, D., Louembet, C., Rondepierre, A., and KaraZaitri, M. (2013). A new mixed iterative algorithm to solve the fuel-optimal linear impulsive rendezvous problem. J. Opt. Theor. Appl., 159, 210-230.

Asawa, S., Nagashio, T., and Kida, T. (2006). Formation flight of spacecraft in earth orbit via MPC. In $S I C E-$ ICASE International Join Conference.

Berkelaar, M., Eikland, K., and Notebaert, P. (2010). Lpsolve, an open source (mixed-integer) linear programming system. http://sourceforge.net/projects/lpsolve/.

Breger, L. and How, J.P. (2008). Safe trajectories for autonomous rendezvous of spacecraft. J. Guid. Contr. Dynam., 31(5), 1478-1489.

Carter, T.E. (1998). State transition matrices for terminal rendezvous studies: Brief survey and new example. J. Guid. Contr. Dynam., 21(1), 148-155.

Clohessy, W.H. and Wiltshire, R.S. (1960). Terminal guidance systems for satellite rendezvous. J. Aerosp. Sc., 27(9), 653-658.

Gavilan, F., Vazquez, R., and Camacho, E.F. (2009). Robust model predictive control for spacecraft rendezvous with online prediction of disturbance bound. In Proceedings of AGNFCS'09, Samara, Russia,.

Gavilan, F., Vazquez, R., and Camacho, E.F. (2012). Chance-constrained model predictive control for spacecraft rendezvous with disturbance estimation. Contr. Eng. Pract., 20(2), 111-122.

Larsson, R., Berge, S., Bodin, P., and Jönsson, U. (2006). Fuel efficient relative orbit control strategies for formation flying and rendezvous within prisma. In Proceedings of the 29th AAS guidance and control conference.

Richards, A.G. and How, J. (2003). Performance evaluation of rendezvous using model predictive control. AIAA Paper 2003-5507.

Rossi, M. and Lovera, M. (2002). A multirate predictive approach to orbit control of small spacecraft. In Proceedings of $A C C$ 2002.

Tschauner, J. and Hempel, P. (1965). Rendevous zu einem in elliptischer bahn umlaufenden. Ziel. Acta Astronaut., II(2), 104-109.

Vazquez, R., Gavilan, F., and Camacho, E.F. (2011). Trajectory planning for spacecraft rendezvous with on/off thrusters. In Proc. of IFAC World Congress 2011.

Wie, B. (1998). Space vehicle dynamics and control. AIAA.

Yamanaka, K. and Ankersen, F. (2002). New state transition matrix for relative motion on an arbitrary elliptical orbit. J. Guid. Contr. Dynam., 25(1), 60-66. 